\title{
Multiply Perspective Polygons inscribed in a Plane Cubic Curve.
}

\author{
By Dr D. G. TaYlor.
}

(Received 29th August 1920. Read 10th December 1920).

Prof. W. P. Milne has cited (Proc. Edin. Math. Soc., Vol. XXXI., p. 90) pairs of nonagons inscribed in a plane cubic curve, and in nonuple perspective. Apart from this, and the familiar case of triangles, * little attention appears to have been given to polygons in multiple perspective.

The use of the term "perspective" here must be noted. Two polygons $A_{1} A_{2} \ldots A_{n}, B_{1} B_{2}, \ldots B_{n}$ are in perspective in the present sense if, for example, the joins $A_{1} B_{1}, A_{2} B_{2}, \ldots A_{n} B_{n}$ are concurrent. In the case of triangles such a relation defines a projective transformation of the whole plane. For $n>3$, this is not necessarily so; and to indicate that the perspective relation is postulated only of two systems of points, we shall speak of an "n-ad of points" (on the analogy of triad, tetrad), instead of an " $n$-gon." The cases with which we shall deal constitute trans. formations of a cubic curve into itself. We shall prove that, for all values of $n$, there exist cases of two $n$-ads in n-ple perspective; or, including in our view the $n$ poles, that there exist cases of three $n$-ads in mutual n-ple perspective; i.e. each pair are in n-ple perspective, and have the points of the third as perspective poles.

\section{Cychio n-ads on a Cubic Curve.}

Let $A_{r}(r=0,1, \ldots n-1)$ be $n$ points on a non-singular plane cubic curve, defined by the elliptic parameters

$$
\alpha+r \Omega / n,(r=0,1, \ldots n-1),
$$

where $\alpha$ is a constant and $\Omega$ a period; they may be said to constitute a cyclic $n$-ad of points on the cubic. If we put

$$
\Omega=2 m \omega+2 m^{\prime} \omega^{\prime},
$$

*See Proc. Edinburgh Maih. Soc., Vol. XXXI., p. 64, and references there given. 
where $2 \omega, 2 \omega^{\prime}$ are the primitive periods, and $m, m^{\prime}$ integers which may take any values from 0 to $(n-1)$ inclusive, we shall clearly have different kinds of cyclic $n$-ads according to the values of $m, m^{\prime}$. When $n$ is prime, the number of different kinds will be $\frac{1}{2}\left(n^{2}-1\right)$; when composite, the formula will be less simple. Thus there are four distinct kinds of triads, corresponding to

$$
\Omega=2 \omega, 2 \omega^{\prime}, 2 \omega+2 \omega^{\prime}, 2 \omega+4 \omega^{\prime} ;
$$

and there are only six distinct kinds of non-degenerate tetrads, corresponding to

$$
\Omega=2 \omega, 2 \omega^{\prime}, 2 \omega+2 \omega^{\prime}, 2 \omega+4 \omega^{\prime}, 4 \omega+2 \omega^{\prime}, 2 \omega+6 \omega^{\prime} .
$$

1. Any two cyclic nads of the same kind are in n-ple perspective, their poles being also on the curve, and constituting a third cyclic n-ad of the same kind.

For, let the $n$-ads and their elliptic parameters be

$$
A_{r}(\alpha+r \Omega / n), B_{r}(\beta+r \Omega / n),(r=0,1,2, \ldots n-1),
$$

where $\alpha, \beta$ are any constants, and consider the third $n$-ad

where

$$
C_{r}(\gamma+r \Omega / n),(r=0,1,2, \ldots n-1)
$$

Clearly $A_{r} B_{t} C_{t}$ are collinear, provided $r+s+t \equiv 0(\bmod n)$. Keeping $t$ fixed, we have corresponding to each value of $r$ in the range prescribed, one, and only one, value of $s$ in the same range.

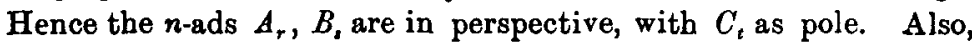
this is true for every $t$ from 0 to $(n-1)$ inclusive.

Since the relation between the $A, B$, and $C n$-ads is mutual, this is a case of three n-ads in mutual n-ple perspective.

Other properties of the cyclic $n$-ad may be noted.

2. The orders of the $B_{r}$ which yield perspective relations with the points $A_{0} A_{1} \ldots A_{n-1}$ are the $n$ cyclic permutations of

$$
B_{n-1} B_{n-2} \ldots B_{1} B_{0} \text {. }
$$

3. The parameters $\alpha, \beta$, and the absolute invariant of the cubic curve being at our disposal, it seems likely that $n$-ads upon the cubic exist which are in perspective in other ways in addition to the $n$ ways just considered. Such additional perspective relations will correspond to a different cyclic arrangement of the points $B_{r}$; and the new perspective poles cannot lie on the cubic. 
4. By marking the points in which the joins of a cyclic $n$-ad meet the curve again, we obtain, in consequence of coincidences, only $n$ distinct points; and these constitute a cyclic $n$-ad of the same kind as the original.

5. When $n$ is odd, the "tangentials" of the points of a cyclic $n$-ad constitute a cyclic $n$-ad of the same kind; indeed, identical with that determined by the joins. When $n$ is even, the tangentials form a degenerate $n$-ad, of $\frac{1}{2} n$ points each counted twice.

\section{Cyclic Triads on the Cubic.}

The case of cyclic triads presents noticeable features. Proofs are simple, and bare statement will suffice.

1. If three triangles are in mutual triple perspective, their vertices are the common points of a pencil of cubic curves; and, with reference to any member of the pencil, constitute three cyclic triads of the same kind.

2. Sextuply Perspective Triangles. The remarkable case of sextuply perspective triangles (see footnote to first paragraph) may be described as follows:

The vertices of the triangle of reference,

$$
X_{1}(1,0,0), X_{2}(0,1,0), X_{3}(0,0,1)
$$

and the three triads

$$
\begin{aligned}
& P_{1}\left(\epsilon^{2}, \epsilon, 1\right), P_{2}\left(\epsilon, \epsilon^{2}, 1\right), P_{3}(1,1,1), \\
& Q_{1}\left(\epsilon^{2}, 1,1\right), Q_{2}\left(1, \epsilon^{2}, 1\right), Q_{3}\left(1,1, \epsilon^{2}\right), \\
& R_{1}(\epsilon, 1,1), R_{2}(1, \epsilon, 1), R_{3}(1,1, \epsilon),
\end{aligned}
$$

where $\epsilon$ is an imaginary cube root of unity, define four triangles such that each pair are in sextuple perspective, with the vertices of the remaining pair as perspective poles. Through any three of these four triads passes a pencil of cubics. Thus through the $X_{r}, P_{r}, Q_{r}$ pass all cubics of the pencil

$$
\left(x_{2}-x_{3}\right)\left(x_{3}-\epsilon^{2} x_{1}\right)\left(x_{1}-\epsilon x_{2}\right)=\lambda\left(x_{3}-x_{1}\right)\left(x_{1}-\epsilon^{2} x_{2}\right)\left(x_{2}-\epsilon x_{3}\right),
$$

and through the $X_{r}, P_{r}, R_{r}$ pass all the cubics of the pencil

$$
\left(x_{2}-x_{3}\right)\left(x_{3}-\epsilon x_{1}\right)\left(x_{1}-\epsilon^{2} x_{2}\right)=\mu\left(x_{3}-x_{1}\right)\left(x_{1}-\epsilon x_{2}\right)\left(x_{2}-\epsilon^{2} x_{3}\right)
$$

where $\lambda, \mu$ are parameters. The $X_{r}, P_{r}, Q_{r}$ form cyclic triads of the same kind with respect to any member of the first pencil; 
the $X_{r}, P_{r}, R_{r}$ form cyclic triads of the same kind with respect to any member of the second pencil; the cyclic order of one of the triads $X_{r}, P_{r}$ being reversed in the passage from one pencil to the other. This accounts for the sextuply perspective relation. This intimate association is the relation which subsists between the four triangles with reference to which the equation of a nonsingular plane cubic assumes canonical form. The twelve sides of these triangles are in fact the lines on which lie, three by three, the nine flexes of the curve

3. Every cyclic triad on a cubic $S$ is apolar, not only to $S$ itself, but to every member of the pencil $S+\lambda H$, defined by $S$ and its Hessian $H$.

This is an immediate consequence of the condition for apolarity.

\section{Special $n$-ads when $n$ is a Square Number.}

1. The nonuply perspective nonads of Milne are distinct from all cases of cyclic nonads given by the theory of section I. of this paper, and point to a special kind of $n$-ads in the cases when $n$ is a square number.

Let $n=v^{2}$, and consider the $n$ points $A_{m, m^{\prime}}$ defined by the elliptic parameters

$$
A_{m, m^{\prime}}\left(\alpha+\frac{2 m \omega}{v}+\frac{2 m^{\prime} \omega^{\prime}}{v}\right),\left(m, m^{\prime}=0,1,2, \ldots v-1\right),
$$

where $\alpha$ is a constant. These form what we may call a special $n$-ad.

For simplicity let $\nu$ be a prime; let

$$
B_{m, m^{\prime}}\left(\beta+\frac{2 m \omega}{\nu}+\frac{2 m^{\prime} \omega^{\prime}}{\nu}\right),\left(m, m^{\prime}=0,1,2, \ldots \nu-1\right),
$$

be a second special $n$-ad; and consider a third,

$$
C_{m, m^{\prime}}\left(\gamma+\frac{2 m \omega}{\nu}-\frac{2 m^{\prime} \omega^{\prime}}{\nu}\right),\left(m, m^{\prime}=0,1,2, \ldots v-1\right),
$$

where $\alpha+\beta+\gamma \equiv 0\left(\operatorname{modd} 2 \omega, 2 \omega^{\prime}\right)$.

If we choose any definite points $A_{r, r^{\prime}}, C_{t, t^{\prime}}$ from the first and third, there is clearly one, and only one, point of the second collinear with these, namely $B_{d, d^{\prime}}$, where

$$
r+s+t \equiv 0, r^{\prime}+s^{\prime}+t^{\prime} \equiv 0(\bmod v) \text {. }
$$


Keeping $t, t^{\prime}$ fixed and permitting $r, r^{\prime}$ to vary, we find that the $n$-ads $A_{m, n^{\prime}}, B_{m, m^{\prime}}$ are in perspective, with $C_{t, t^{\prime}}$ as pole. But since this holds for all values of $t, t^{\prime}$, the n-ads $A_{m, m^{\prime}}, B_{n, m^{\prime}}$ are in n-ple perspective, with the points $C_{m, m^{\prime}}$ as poles.

2. It is easy to distinguish this type of $n$-ply perspective conformation from that associated with cyclic $n$-ads. In the case of cyclic $n$-ads, the arrangements of the $B$-points corresponding to a fixed order of the $A$-points in the $n$ perspective relations (I., 2 above) preserve the same cyclic order of the B-points. In the case of special $n$-ads, the B-points change cyclically in groups of $v$ each. Thus for the poles $C_{0,0}, C_{1,0}$, the arrangements of the $B$-points corresponding to the given order of $A$-points are as follows:

$$
\begin{aligned}
& A_{0,0}, A_{1,0}, A_{2,0} ; A_{0,1}, A_{1,1}, A_{2,1} ; A_{0,2}, A_{1,2}, A_{2,2} ; \\
C_{00}: & B_{0,0}, B_{2,0}, B_{1,0} ; B_{0,2}, B_{2,2}, B_{1,2} ; B_{0,1}, B_{2,1}, B_{1,1} ; \\
C_{1,0}: & B_{2,0}, B_{1,0}, B_{0,0} ; B_{2,2}, B_{1,2}, B_{0,2} ; B_{2,1}, B_{1,1}, B_{0,1} .
\end{aligned}
$$

3. The simplest cases of special $n$-ads occur when $n=4,9$. In the former case, we obtain tetrads with elliptic parameters of the form

$$
\alpha, \alpha+\omega, \alpha+\omega^{\prime}, \alpha+\omega+\omega^{\prime},
$$

of which the properties are well known.* In the latter case we obtain nonads defined by

$$
\alpha+\frac{2 m \omega}{3}+\frac{2 m^{\prime} \omega^{\prime}}{3},\left(m, m^{\prime}=0,1,2\right) .
$$

Of these the 9 flexes are an example; while, as shown by Milne, the intersections of the cubic $S$ with any member of the pencil of class cubics defined by $P$ the Cayleyan of $S$, and the Hessian of $P$, constitute two nonads of this type, which, with the flexes, form three nonads in mutual $n$-ple perspective.

"See a paper by Milne, Proc. Edinburgh Math. Soc., Vol. XXIX, p. 2. 